\title{
Review: Proximity effects in the production of chromosome aberrations by ionizing radiation
}

\author{
R. K. SACHS $\dagger^{*}$, A. M. CHEN $+\ddagger$ and D. J. BRENNER \\ (Received 4 July 1996; accepted 10 September 1996)
}

\begin{abstract}
After ionizing radiation has induced double-strand DNA breaks (dsb), misrejoining produces chromosome aberrations. Aberration yields are influenced by 'proximity' effects, i.e. by the dependence of misrejoining probabilities on initial dsb separations. We survey proximity effects, emphasizing implications for chromosome aberration-formation mechanisms, for chromatin geometry, and for dose-response relations. Evidence for proximity effects comes from observed biases for centric rings and against three-way interchanges, relative to dicentrics or translocations. Other evidence comes from the way aberration yields depend on radiation dose and quality, tightly bunched ionizations being relatively effective. We conclude: (1) that misrejoining probabilities decrease as the distance between dsb at the time of their formation increases, and almost all misrejoining occurs among dsb initially separated by $<1 / 3$ of a cell nucleus diameter; (2) that chromosomes occupy (irregular) territories during the $\mathrm{G}_{0} / \mathrm{G}_{1}$ phase of the cell cycle, having dimensions also roughly $1 / 3$ of a cell nucleus diameter; (3) that proximity effects have the potential to probe how much different chromosomes intertwine or move relative to each other; and (4) that incorporation of proximity effects into the classic random breakage-and-reunion model allows quantitative interrelation of yields for many different aberration types and of data obtained with various FISH painting methods or whole-genome scoring.
\end{abstract}

\section{Introduction}

Subjecting cells to ionizing radiation during the $\mathrm{G}_{0} / \mathrm{G}_{1}$ phase of the cell cycle causes chromosometype aberrations, through chromosome breakage and large-scale rearrangement of the pieces. At various times, chromosome aberrations have been suggested as symptoms and/or causes of most major radiobiological effects (survey in Cornforth and Bedford 1993). They are of particular in terest in connection with biodosimetry (e.g. Bender et al. 1988, Lucas et al. 1992, Bauchinger 1995, Durante

*Author for correspondence: R. K. Sachs.

†Department of Mathematics, University of California, Berkeley, CA 94720, USA. sachs@math.berkeley.edu

¡Lawrence Livermore National Laboratory, PO Box 808, Livermore, CA 94551, USA.

$\S$ Center for Radiological Research, Columbia University, New York, NY 10032, USA. et al. 1996, Gebhart et al. 1996) or as indicators of radiosensitivity (e.g. Wlodek and Hittelman 1988, Jones et al. 1995, Russell et al. 1995). Certain chromosome aberrations are strongly linked with most haematopoietic cancers (Rabbitts 1994).

It is probable (Cornforth and Bedford 1993; see also $\$ 2$ ) that most chromosome aberrations result from illegitimate reunion ('misrejoining') of free ends from different DNA double-strand breaks (dsb). Accepting this picture, one finds that aberration formation is influenced by 'proximity' effects, i.e. effects which occur because dsb free ends are more likely to undergo illegitimate reunion if the $\mathrm{dsb}$ are initially formed close together than if the $\mathrm{dsb}$ are formed far apart (Sax 1940, survey in Savage 1996). Proximity effects can be inferred by analysing aberration yields as a function of aberration type, of radiation quality, or of dose.

Proximity effects influence relative yields of different types of aberrations, because any one chromosome at one time is somewhat localized in an irregular territory (or 'domain') during $\mathrm{G}_{0}$ / $G_{1}$, rather than being spread out more or less uniformly over the whole cell nucleus (Appendix 2). For example, compared with expectations based on complete randomness, proximity effects bias for centric rings (which involve two dsb on one [localized] chromosome) relative to dicentrics (which involve two dsb on two different chromosomes). It is remarkable that chromosome aberration data suggested reasonable models for chromosome localization (Sax 1940, Savage and Papworth 1973) long before modern methods gave direct confirmation that chromosomes do occupy localized territories during $\mathrm{G}_{0} / \mathrm{G}_{1}$ (Appendix 2).

Proximity effects influence relative aberration yields for radiations of different quality inasmuch as high LET radiations producing tightly bunched $\mathrm{d} s \mathrm{~b}$ are more effective than low LET radiations in producing aberrations (survey in Goodhead 1987). Proximity effects also enhance the importance of the nearby dsb pairs induced by a single primary radiation track, compared with the relatively 
distant $\mathrm{dsb}$ pairs induced by different primary radiation tracks, an effect which can be uncovered by varying the dose (survey in Kellerer 1985).

The advent of fluorescent in situ hybridization (FISH) chromosome painting has dramatically increased the scope of aberration studies (survey in Gray et al. 1994, Simpson and Savage 1996). Detailed results on many types of simple or complex aberrations have been obtained, and more sophisticated painting techniques (Ballard and Ward 1993) promise to reveal additional aspects of aberrations and proximity effects.

We shall here review some current ideas on proximity effects. Damage leading to loss or transfer of chromosome portions $<1 \mathrm{Mb}\left(10^{6}\right.$ base pairs) will not be considered directly, since the resolution afforded by light microscopy is not adequate for routine detection of such 'small' scale phenomena. Moreover, to keep the review focused, no detailed discussion of related endpoints such as chromatid aberrations, clonogenic inactivation, mutations or length distributions of broken DNA fragments will be given; some premature chromosome condensation (PCC) results will be discussed, but only those on the kinetics of aberration formation. Section 2 outlines some of the background, the terminology, and the models needed to interpret chromosome aberration data. Section 3 concerns evidence for proximity effects, based on the relative frequencies of particular aberration types, on the relative effectiveness of different qualities of radiation, on the dose dependence of aberration formation, and on cell-to-cell variation of aberration number. Section 4 briefly discusses the relations of proximity effects to the kinetics of aberration formation. After summarizing the results, $\S 5$ discusses some implications for cell nucleus ultrastructure and for applications. Three appendices respectively discuss: (a) randomness of $d s b$ induction and of $d s b$ free end illegitimate reunion; (b) current information on large-scale chromosome geometry and motion; and (c) dose-response relations.

Many of the basic ideas throughout this review were first clarified by D. E. Lea, more than a halfcentury ago (Lea 1946).

\section{Prologue: aberration types and aberration formation mechanisms}

Understanding and organizing data on chromosome aberrations and analyzing proximity effects coherently requires som e conceptual model, explicit or implicit, of how aberrations are formed.
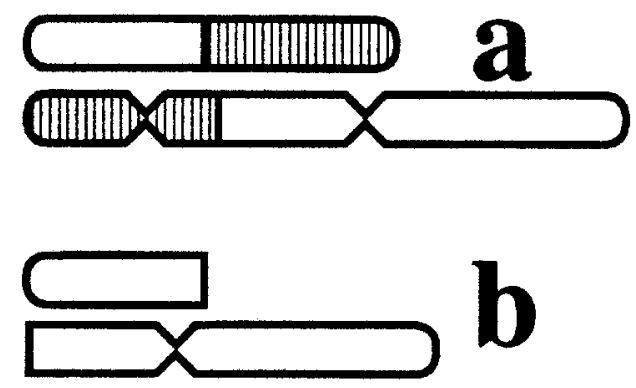

Figure 1. Basic aberration types. Chromosome centromeres are shown as constrictions; telomeres are shown rounded, and are assumed non-reactive (not 'sticky'). (a) An example of an aberration involving two different chromosomes. The simplest interpretation is that just after irradiation each chromosome contained a $\mathrm{dsb}$ and there was subsequent illegitim ate reunion to produce the pattern shown; Figure $2 \mathrm{a}$ and $\mathrm{b}$ show this interpretation more explicity. The aberration in (a) is called 'exchange-type' because the simplest in terpretation is that more than one dsb was involved, is called an 'interchange' because more than one chromosome is involved, and is called a 'dicentric' because one of the two rearranged chromosomes now has two centromeres. (b) A single broken chromosome; this aberration is not exchange-type because a single unrejoined dsb could produce the aberration. Exchange-type aberrations are the most commonly observed chromosome aberrations and are the aberrations of interest in the present paper.

\subsection{Exchange-type aberrations}

The initial chromosome 'lesions' which lead to aberrations are here identified with $\mathrm{dsb}$, as is now fairly standard (Savage 1996). The most commonly observed chromosome aberrations are 'exchange-type' aberrations, i.e. aberrations that appear to result from illegitimate reunions involving two or more dsb. Figure 1a shows a simple example. The conventional view has long been (e.g. Lea 1946) that at least two radiation-induced breaks are actually involved in an exchange-type aberration, as suggested by: (a) the visual appearance of such aberrations; (b) the shape of doseresponse curves; and (c) the influence of low dose-rate or split-dose irradiation on aberration yields. This conventional view has sometimes been challenged, in favour of a model where a single radiation-induced lesion initiates molecular reactions, which eventually lead to an exchange-type aberration (e.g. Chadwick and Leenhouts 1981, Goodhead et al. 1993). However, the conventional view has gained powerful support from an experiment by Cornforth (1990), who looked at exchange-type aberrations produced when pairs of cells were virally fused together. The yield of aberrations involving genomic material from 

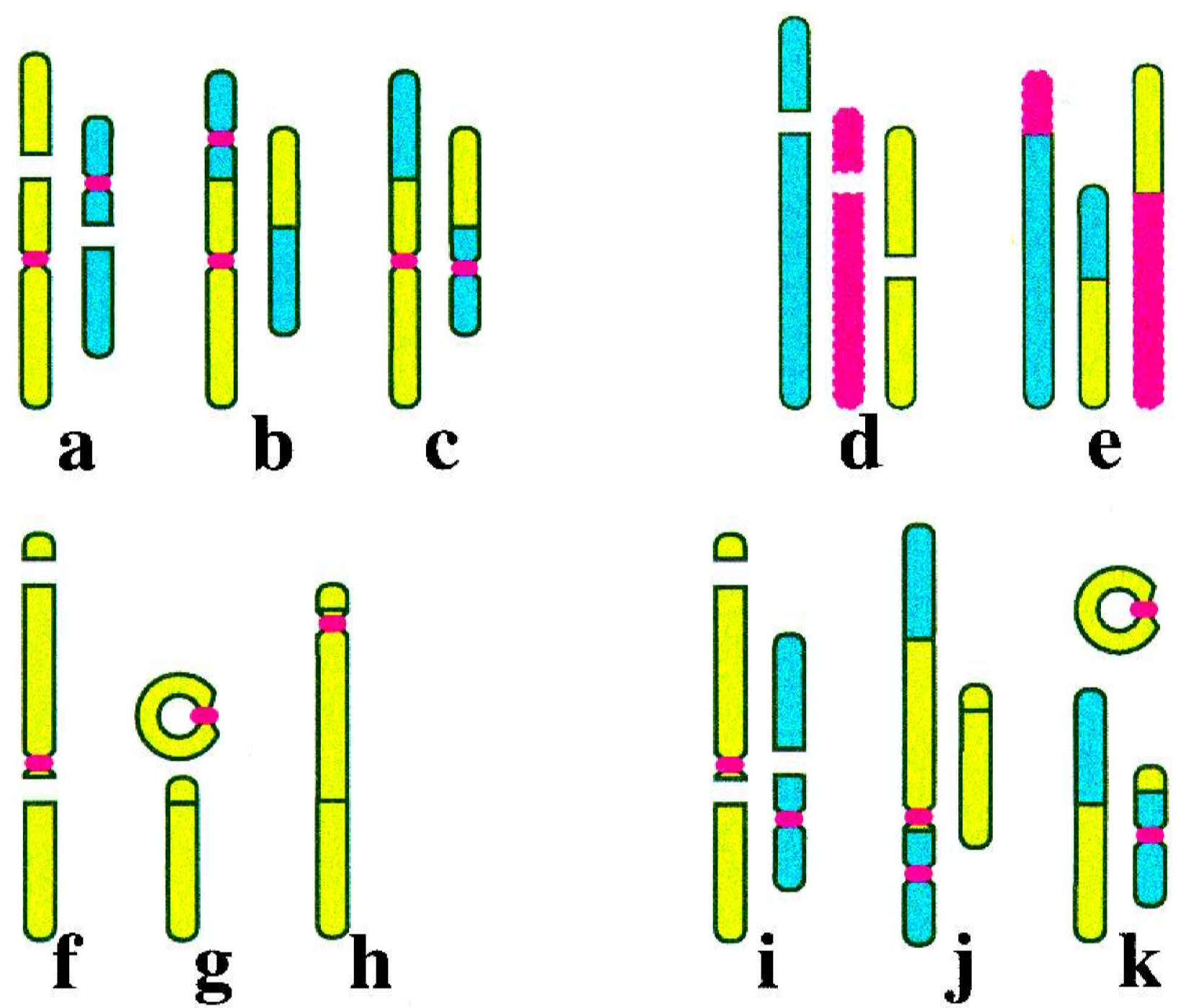

Figure 2. Exchange-type aberrations. (a-c) Apparently simple two-way in terchanges. We show one chromosome painted yellow, one chromosome counter-stained blue, and centromeres painted red. Suppose that there are two reactive dsb, shown as gaps in (a), with four corresponding reactive free ends. Then one possible outcome of illegitim ate reunion is a 'dicentric' (b) and another possible outcome is a 'translocation' (c). The outcomes shown in (b) or (c) are both called 'apparently simple' meaning the final pattern of colours and centromeres could have resulted from in teractions of just two dsb on two different chromosomes (Savage and Sim pson 1994). In the CAB classification of Savage and Simpson (1994), (b) is type 2A and (c) is type 2B. (d and e) Three-way in terchanges. Suppose that there are three chromosomes, each having one reactive $\mathrm{dsb}(\mathrm{d})$. Then one possible outcome of reunion is a three-way interchange (e). This outcome is called 'complex' (Savage and Simpson 1994), i.e. three or more dsb on two or more chromosomes were involved according to the breakage-andreunion model. (e) has eight subcases, differing only in their centromere arrangement. For example, one subcase has one centromere on each rearranged chromosome, i.e. is $\mathrm{t}(\mathrm{Ab}), \mathrm{t}(\mathrm{Bc}), \mathrm{t}(\mathrm{Ca})$ in the classification of Tucker et al. (1995a). Even with a painting system such as that shown in $(\mathrm{a}-\mathrm{c})$ instead of the painting system shown in (d and e), certain three-way interchanges can be identified by their colour/centromere pattern (Brown and Kovacs 1993, Savage and Simpson 1994). (f-h) Intra-chromosomal aberrations. (f) Two reactive dsb; (g) One of the possible outcomes, a centric ring. (h) Another possible outcome, called a pericentric inversion. Corresponding 1 -arm forms (not shown) are acentric rings and paracentric inversions. (i-k) Like the three-way interchange of (e), the patterns shown in (j) (an insertion) and (k) are visibly complex aberrations.

different cells was large when irradiated cells were fused with other irradiated cells, but very much smaller when irradiated cells were fused with unirradiated cells. This result strongly supports, for the large majority of exchange-type aberrations, the conventional view that the aberrations involve two or more radiationinduced breaks; this view will therefore be adopted throughout the rest of this review, although it is certainly possible that some exchange-type aberrations are formed by other mechanisms. 


\subsection{The breakage-and-reunion model}

There is substantial recent evidence (e.g. Brown et al. 1993, Lucas and Sachs, 1993, Chen et al. 1996, Simpson and Savage 1996) that the classic breakage-and-reunion model (Sax 1940, Lea 1946, Savage 1996) gives useful approximations for the frequency of various exchange-type aberrations. A version of the model, mathematically equivalent to a version described by Lea (1946, pp. 166-7, with $q=1$ ) but visualized in terms of $\mathrm{dsb}$, involves three steps as follows. The first step of aberration formation is that ionizing radiation induces multiple $\mathrm{dsb}$. These $\mathrm{dsb}$ are perhaps best pictured as temporarily 'splinted', i.e. the two free ends of a dsb are held close to each other, possibly by proteins (Savage 1996). The next step is that most of the dsb are systematically 'restituted' (i.e. repaired, except perhaps for damage too smallscale for the assay to uncover), while the other dsb become 'reactive' instead of restituting. Reactive $\mathrm{dsb}$ can be visualized as dsb whose free ends have moved apart, and it is these reactive $d s b$, which are to be identified with Lea's 'breaks'. The third and final step is 'illegitimate reunion or accidental restitution': free ends of reactive dsb gradually undergo pairwise reunion, and unless a free end happens to undergo reunion with its own partner ('accidental restitution'), the reunion is illegitimate, causing a chromosome aberration. The number of dsb initially formed by radiation, probably $>40$ per Gy for human cells (Goodhead 1987), is much larger than the number of $d s b,<2$ at $1 \mathrm{~Gy}$, whose free ends ultimately participate in observed illegitimate reunions.

In the simplest form of the breakage-andreunion model, all free $\mathrm{dsb}$ ends do eventually undergo restitution or illegitimate reunion. However, experiments show that a full treatment should include 'incomplete' outcomes, i.e. a few free ends remain unrejoined (for recent surveys see Cornforth and Bedford 1993, Hahnfeldt et al. 1995, Simpson and Savage 1996, Wu et al. 1996b). Figure $1 \mathrm{~b}$ is an example of an incomplete outcome. The model can be extended to include some incomplete outcomes in various slightly different ways (Lea 1946, Hahnfeldt et al. 1992), but this review will emphasize complete outcomes.

\subsection{Aberration types}

For analysis of chromosome aberrations using conventional brightfield microscopy rather than the newer FISH technique a common approach is to determine the average number of dicentrics (Figure 2b) per cell. Another commonly scored category is rings, especially centric rings (Figure $2 \mathrm{~g}$ ). Accurate scoring of translocations (Figure 2c), inversions (e.g. Figure 2h), and some other aberrations using conventional microscopy typically involves detailed banding analysis (e.g. Ohtaki 1992).

In the FISH technique, a subset of chromosomes is fluorescently painted, and only aberrations involving these fluorescently painted chromosomes are scored. A rich and colourful spectrum of aberration types can be analysed rapidly, considerable specialized terminology being needed for describing the various aberration types (Savage and Simpson 1994, Tucker et al. 1995a). Figure 2 shows a few representative aberration types and several representative FISH painting schemes. What is actually scored are the final products (Figure 2b, c, e, g, h, j or k), the putative formation mechanisms given in the figure caption being model-dependent. Not all outcomes of the initial break patterns are shown. For example, according to the breakage-and-reunion model, the situation shown in Figure 2a can also lead to accidental restitution of both chromosomes or various incomplete patterns resulting from the failure of two or four free ends to rejoin.

\subsection{Randomness}

There is evidence (Appendix 1) for randomness of breakage, i.e. that any long stretch of DNA has about the same chance of containing a radiationinduced reactive $\mathrm{dsb}$ as any other equally long stretch (with length measured in $\mathrm{Mb}$ ). There is also evidence for randomness of illegitimate reunion, i.e. that the reunion probability of two free ends depends only on their initial spatial separation (Appendix 1). As Lea (1946) showed, randomness assumptions transform the breakageand-reunion model from a qualitative picture to a quantitative theory, capable of making numerical predictions about relative yields for many different types of aberrations. Probably randomness is violated to some extent, but it is a useful first approximation, and specific observable deviations from randomness can be identified by working out the mathematical consequences of randomness in detail. It is also possible to use the breakageand-reunion model with partial randomness assumptions, i.e. randomness assumptions less far reaching than those described in Appendix 1 (e.g. Simpson and Savage 1996). 


\subsection{An alternative model}

One alternative to the breakage-and-reunion model is a model in which pairwise illegitimate reunion of two free ends from two dsb implies that the two remaining free ends from the same two dsb must join with each other (or remain permanently unrejoined, resulting in an incomplete outcome). This alternative model can be motivated, at least in part, by the exchange-first hypothesis of Revell (described in Revell 1974, Savage 1996). However, substantial yields are found of aberrations impossible or difficult to produce according to the alternative model (Brown and Kovacs 1993, Lucas and Sachs 1993, Savage and Simpson 1994, 1996, Chen et al. 1996), for example three-way interchanges (Figure 2e) and certain insertions (e.g. Figure 2j). In addition, when tracking illegitimate reunions in time, they are found to form singly, not in pairs, a result hard to reconcile with the alternative model (Brown et al. 1993).

\subsection{Sites and interaction distances}

Proximity effects can typically be incorporated into a pairwise interaction model (such as the breakage-and-reunion model, the alternative model of the preceding subsection, or the Theory of Dual Radiation Action) in either of two related ways: using 'sites' or using 'interaction distances' (Kellerer and Rossi 1978). Site models are often less realistic than interaction distance models but usually involve fewer adjustable parameters.

Conceptually, sites are subregions of the cell nucleus within which interactions can take place (survey in Savage 1996). For example, one can incorporate proximity effects in to the breakageand-reunion model by dividing the nucleus into sites, and assigning chromosomes at random to the sites, with illegitimate reunion possible between $d s b$ free ends only if the $d s b$ are in the same site (Chen et al. 1996). When using sites, proximity effects are quantified by giving the number of sites per cell nucleus or giving the average size of a site.

Interaction distance models, on the other hand, focus on the separation between two dsb (or two $\mathrm{dsb}$ free ends) at the time of $\mathrm{dsb}$ formation. Proximity effects are quantified by giving reunion probabilities as a function of this interaction distance (Kellerer and Rossi 1978). For a sphere of $6 \mu \mathrm{m}$ diameter (a typical cell nucleus size which we shall use for reference throughout though it is more appropriate for human lymphocytes than for human fibroblasts) random point pairs are on average just $>3 \mu \mathrm{m}$ apart; maximum interaction distances $<6 \mu \mathrm{m}$ or average interaction distances $<3 \mu \mathrm{m}$ indicate proximity effects.

\section{Evidence for proximity effects}

\subsection{Evidence from relative frequencies of aberration type}

Figure $2 \mathrm{a}-\mathrm{h}$ shows aberrations involving different numbers of chromosomes, from one to three. As we will now discuss, proximity bias yields of these aberrations toward aberrations involving fewer chromosomes.

3.1.1. Centric rings. For aberrations produced by ionizing radiation, it has long been known (e.g. Sax 1940, Savage 1970, Hlatky et al. 1992) that the observed ratio of dicentrics (Figure $2 b$ ) to centric rings (Figure $2 \mathrm{~g}$ ), designated $D / R$, is much smaller than would be expected if randomness held without any spatial effects. The discrepancy has traditionally been interpreted as evidence for chromosome localization and for proximity effects: if chromosomes are localized in territories, then two dsb on opposite arms of one chromosome are on average closer together than two dsb on two different chromosomes, and proximity effects would thus lead to a bias for illegitimate rejoining in the one-chromosome (ring) case compared with the two-chromosome (dicentric) case, as observed.

For complete randomness (as described in Appendix 1), and small doses, the $D / R$ ratio in a cell having $4 n$ chromosome arms all of the same length should be $D / R=4 n-2$ if there were no proximity effects. For example in a human cell $2 n=46$, so if all chromosome arms were equally long the predicted ratio would be $D / R=$ $4 \times 23-2=90$. This number can be understood as follows. Consider a reactive $\mathrm{dsb}$ on one arm of one chromosome. Suppose, at low dose, there is just one other reactive $d s b$. There are 92 arms on which this second dsb can be located. If it is on the opposite arm of the same chromosome there is a $50 \%$ chance illegitimate reunion would result in a centric ring (Figure $2 \mathrm{~g}$ ). If it is on any of 90 other arms there is a corresponding $50 \%$ chance of a dicentric (Figure 2b). Thus, assuming randomness and all arm lengths equal, the $D / R$ ratio should be 90 . This argument can readily be corrected for the fact that different chromosomes have different arm lengths. Let $L_{i}$ be the length of the long arm of the $i$ th chromosome, $S_{i}$ be the length of 
the short arm. Then, extending the argument just given (Appendix 1), randomness would give (Savage and Papworth 1982, Hlatky et al. 1992)

$$
D / R=\frac{\sum_{i=1}^{46}\left(L_{i}+S_{i}\right)\left(G-L_{i}-S_{i}\right)}{2 \sum_{i=1}^{46} L_{i} S_{i}}=86 .
$$

Here 86 comes from the specific human chromosome arm lengths averaged over males and females (Sachs et al. 1993). The predicted ratio is independent of dose, provided the dose is so small that complex aberrations (e.g. Figure $2 \mathrm{e}$ or $\mathrm{j}$ ) do not play a significant role. At higher doses, Monte Carlo computer simulations (Chen et al. 1996) can calculate the required corrections to equation 1 . Assuming randomness (Appendix 1), equation 1 also applies to the ratio of translocations (Figure 2c) to pericentric inversions (Figure $2 \mathrm{~h}$ ); in general the ratio of inter-chromosomal aberrations (Figure 2b or c) to corresponding inter-arm aberrations (Figure $2 \mathrm{~g}$ or $\mathrm{h}$ ) is designated as $F$ (Hlatky et al. 1992, Brenner and Sachs 1994a).

Experimentally, for sparsely ionizing radiation, $D / R$ consistently comes out in the range $5-20$, with mean 16 (Hlatky et al. 1992), rather than the 86 predicted by equation 1. A number of chromosome geometry models interpret the smaller experimental ratio in terms of proximity effects (Savage and Papworth 1973, Hlatky et al. 1992, Brenner et al. 1994, Chen et al. 1996, Savage and Papworth 1996). For example, using a site model Chen et al. (1996) found by computer simulations $D / R \approx 84 / S$, where $S$ is the number of sites in the cell nucleus, so that the $D / R$ observations suggest site numbers in the range $4-17$. The various models that have been used to analyse the data generally agree on three points; (a) a chrom osome occupies a territory with volume very roughly $(1 / 3)^{3}$ of the cell nucleus volume; (b) free ends from dsb initially formed as much as $\sim 2 \mu \mathrm{m}$ apart can apparently interact (so that tight, time-independent chromosome binding to a rigid nuclear skeleton seems unlikely); and (c) proximity effects must be present, preventing or strongly inhibiting interactions of dsb free ends initially more than $\sim 2 \mu \mathrm{m}$ apart. The models disagree on the amount of chromosome overlapping and intertwining, and on the extent to which a free dsb end on one chromosome can move through or around other chromosomes to reach a different free dsb end.

The question of how $D / R$, and more generally $F$, depends on LET has recently been the subject of debate (Savage and Papworth 1996, Brenner and Sachs 1996a, b, Schmid and Bauchinger 1996). Brenner and Sachs (1994a) proposed that $F$ has the potential to act as a fingerprint of previous exposure to low doses of densely-ionizing radiation (e.g. Brenner 1996). They suggested that a single track of densely ionizing radiation might be expected to produce an extra bias towards interarm exchanges (centric rings or pericentric inversions) relative to inter-chromosomal exchanges (dicentrics or translocations). This is because the closer proximity of chromosomal breaks produced by a single high-LET track would be expected to result in more dsb within a given chromosome, compared to a situation where dsb are spatially uncorrelated, and therefore randomly located. The resulting bias towards inter-arm aberration formation by high-LET radiation would be expected to hold in a situation where chromosomes are localized, and chromosome free ends can undergo relatively unobstructed motion over large distances. If, by contrast, large-scale motion is highly restricted, such LET effects might not occur (Savage and Papworth 1996).

In approaching this question experimentally, it is important to note that as the high-LET dose increases to a level where multiple high-LET tracks are produced within a nucleus, the spatial distribution of chromosome breaks will start to resemble that from low-LET radiation. Consequently, a significant LET dependence for $F$ would be most likely to be seen in experiments involving low doses of high-LET radiation, where the average number of high-LET tracks traversing the nucleus is $<1$, which is also the region of interest in environmentally relevant situations. At such low doses of high LET radiation, a significantly low $F$ has been observed (Pohl-Ruling et al. 1986), though at higher doses smaller, and occasionally no, LET dependencies have been observed (e.g. Schmid and Bauchinger 1996).

Analogous to a possible excess of centric rings at high LET, there is some evidence that insertions, which also involve two dsb on a single chromosome, may be more frequent, relative to other complex aberrations, at high LET (Griffin et al. 1995).

\subsubsection{Three-way interchanges. Recent FISH results} have provided new evidence for proximity effects. Just as proximity biases for on e-chromosome aberrations such as rings (Figure $2 \mathrm{~g}$ ) relative to two-way interchanges (Figure $2 \mathrm{~b}$ and $\mathrm{c}$ ), it biases against three-way interchanges (Figure $2 \mathrm{e}$ ) relative to twoway interchanges. For example, in a site model (see $\S 2.6)$, some sites will contain only two chromosomes, and in those sites three-way interchanges are impossible altogether, biasing against 
Table 1. Proximity effects on three-way interchanges.

\begin{tabular}{|c|c|c|c|c|c|c|}
\hline \multirow[b]{3}{*}{ Type } & \multirow[b]{3}{*}{ Obs } & \multicolumn{2}{|c|}{$4 \mathrm{~Gy}$} & \multicolumn{3}{|c|}{$6 \mathrm{~Gy}$} \\
\hline & & \multicolumn{2}{|c|}{ Simulated } & \multirow[b]{2}{*}{ Obs } & \multicolumn{2}{|c|}{ Simulated } \\
\hline & & Prox & Non & & Prox & Non \\
\hline AS & 964 & 964 & 964 & 564 & 564 & 564 \\
\hline $2 \mathrm{~F}$ & 81 & 84 & 348 & 61 & 69 & 240 \\
\hline $2 \mathrm{G}$ & 83 & 78 & 379 & 66 & 63 & 266 \\
\hline $\mathrm{OC}$ & 164 & 121 & 192 & 197 & 156 & 301 \\
\hline
\end{tabular}

'Obs' refers to data from Simpson and Savage (1996). Aberration types are: apparently simple interchanges ('AS'; see Figure 2b and 2c); two kinds of three-way interchanges (Figure 2e), differing in their centromere pattern, and respectively denoted ' $2 \mathrm{~F}$ ' or ' $2 \mathrm{G}$ ' in the CAB terminology of Savage and Simpson (1994); and other complex aberrations ('OC'). Simulation is with a Monte Carlo site model that assumes randomness (Chen et al. 1996) and has its adjustable parameter normalized to give the observed number of apparently simple interchanges. The 1-site, non-proximity simulation ('non') grossly overestimates the number of three-way interchanges. A 10-site proximity simulation ('prox') gives acceptable results (although OC is underestimated).

three-way interchanges relative to two-way interchanges for the cell nucleus as a whole.

FISH experiments do indeed show that the number of three-way interchanges is markedly smaller than a non-proximity calculation assuming randomness would predict. Because the three-way interchanges are complex and compete with many other possible endpoints (Savage and Simpson 1994) there is no simple formula analogous to equation 1 for predicting the relative numbers of three-way interchanges compared to simple interchanges, but Monte Carlo computer simulation techniques can generate the required estimates (Chen et al. 1996). For example, in Table 1, a nonproximity random breakage and reunion model grossly overestimates the number of three-way interchanges compared to experimental results of Simpson and Savage (1996). However, a corresponding proximity model with 10 sites gives approximately the correct number of three-way interchanges.

Other data sets (e.g. Chen et al. 1996) show this same trend. Significant yields of three-way interchanges are observed (Brown and Kovacs 1993, Lucas and Sachs 1993), a result which favours the breakage-and-reunion model over the alternative model described in $\S 2.5$. However, the frequency of three-way interchanges is less than complete randomness would indicate, suggesting that it is difficult for three different chromosomes to come together, as one would expect if chromosomes are localized and free end motion is limited. Quantitatively, computer simulations indicate an average pairwise interaction distance (see $\S 2.6$ ) of $\sim 1.3 \mu \mathrm{m}$ (Chen et al. 1996), generally consistent with the distance inferred from low LET $D / R$ data on dicentrics and centric rings (see $\$ 3.1 .2$ ).

3.1.3. Intra-arm exchanges. Additional evidence for proximity effects is that the yield of 'interstitial deletions', many of which are presumably small acentric rings, is larger than randomness would indicate, even compared to other intrachromosomal aberrations such as centric rings (e.g. Savage 1975, Cornforth and Bedford 1987, Hahnfeldt et al. 1995, Pandita et al. 1995, and references quoted in these papers). Moreover, the frequency of inversions is comparable to the frequency of rings (Muhlmann-Diaz and Bedford 1995) so the frequency of paracentric inversions, which are exchange-type aberrations within a single chromosome arm, is probably comparable with that of acentric rings. Judged from models of chromatin geometry (Appendix 2), the data on acentric rings and inversions imply proximity effects on a length scale substantially smaller than $1 \mu \mathrm{m}$ for dsb pairs located on the same chromosome arm (Wu et al. 1996b).

3.1.4. Restitution. A large majority of the dsb initially induced by ionizing radiation are restituted (see $\S 2.2$ ). This fact may be attributable to proximity effects, at the nanometer level, if restitution and illegitimate reunion are similar molecular events, with the former favoured because the two free ends from a single dsb are, on average, initially much closer than free ends from different dsb (Wu et al. 1996a).

\subsection{Evidence from experiments with differing types of radiations}

Lea (1946) commented that 'study of the relative efficiency of radiations of different ion density in producing structural changes in chromosomes is an important method of attack on the mechanism of this process'. We now summarize some of the results on proximity obtained by such studies. Different types of radiations may differ somewhat in the number and type of dsb they initially induce (Brenner and Ward 1992, Goodhead 1994), but we are here concerned, instead, with proximity effects for interactions involving two dsb.

The experiments discussed in $\S 3.1$ typically involve low LET and comparatively high dose. The proximity effects inferred therefore primarily concern 'inter-track action', i.e. concern interactions 
of $d s b$ from independent primary radiation tracks. Such dsb are randomly located with respect to each other, and their average pairwise initial separation is roughly $3 \mu \mathrm{m}$, so the proximity effects discussed up to now concern primarily micrometer scales. Many of the following arguments, on the other hand, concern 'intra-track' action, i.e. the dsb involved are induced by the same primary radiation track. Such $\mathrm{dsb}$ are correlated and for many radiations are, on average, considerably closer, thus probing proximity effects at the sub-microm eter level.

3.2.1. Soft X-rays. It was also Lea (1946) who pointed out that low-energy X-rays, which interact with biological matter primarily by producing short-ranged secondary electrons, might be excellent probes of aberration formation mechanisms. For example $1.5 \mathrm{keV}$ X-rays typically produce one $0.5 \mathrm{keV}$ and one $1.0 \mathrm{keV}$ electron with a combined range of about $70 \mathrm{~nm}$, while a $270 \mathrm{eV}$ X-ray produces a single photoelectron with a range of about $7 \mathrm{~nm}$. For such ultrasoft X-rays, doses of the order of $1 \mathrm{~Gy}$ produce some tracks containing pairs of closely spaced dsbs, allowing estimates of shortranged proximity effects. Although Catcheside and Lea published data on the biological effects of 1.5 and $3 \mathrm{keV}$ X-rays (summarized in Lea 1946 , pp. 249-52), it appears that their results were probably unreliable, and the first modern data with soft X-rays were published by Neary and colleagues (1964). Subsequently (survey in Goodhead et al. 1993) extensive data have been obtained with $1.5 \mathrm{keV}$ and $270 \mathrm{eV}$ X-rays.

The overall results of this work suggest that for X-rays producing nanometer-range electrons, the lower the photon energy, i.e. the shorter the electron track, the larger the aberration yield. In the context of dsb-dsb interaction models, the markedly enhanced aberration yield (see, for example, Figure 3) is evidence for increasing proximity effects, i.e. increasing interaction probabilities for nearby dsbs, as the distance between dsbs increases from about $100 \mathrm{~nm}$ to $<10 \mathrm{~nm}$, although some of the increase could also be due to an increased yield of dsbs.

3.2.2. Correlated ion pairs. Molecular ion experiments (Kellerer et al. 1980, Geard 1985) constitute a second, conceptually similar, approach to probe proximity effects. In these experiments, exposures were performed with correlated pairs of hydrogen ions, whose mean separation could be varied over sub-micrometer dimensions. Consistent with the results of the soft X-ray experiments, as the average

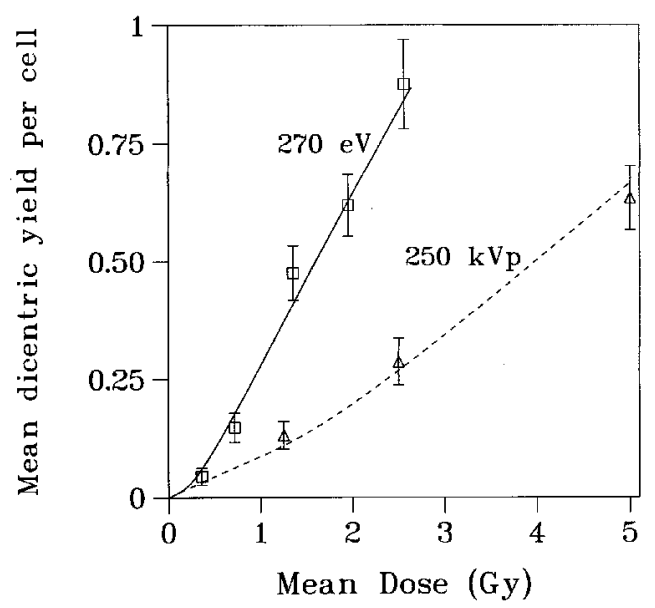

Figure 3. Yield of dicentric chromosome aberrations in V79 hamster cells exposed to graded doses (averaged across the nucleus) of $250 \mathrm{kVp}$ X-rays or ultrasoft $270 \mathrm{eV}$ X-rays. Data from Thacker et al. (1986) and from Thacker (private communication, 1987).

separation between the pairs of ions decreased, the aberration yield increased (Figure 4), again indicating an enhanced proximity effect at submicrometer separations.

\subsubsection{Low-energy neutrons. Both soft X-rays and} molecular ions suffer from difficulties in probing spatial separations below $\sim 50 \mathrm{~nm}$. X-rays of the appropriate energy are strongly attenuated across a cell, while molecular ions suffer multiple scattering. A third category of radiations which have the potential to illuminate the situation are low-energy neutrons; as the neutron energy decreases, the energy of the recoil proton decreases and the range decreases to nanometer dimensions, in analogy to the secondary electrons produced by soft X-rays. Without enhanced proximity effects at

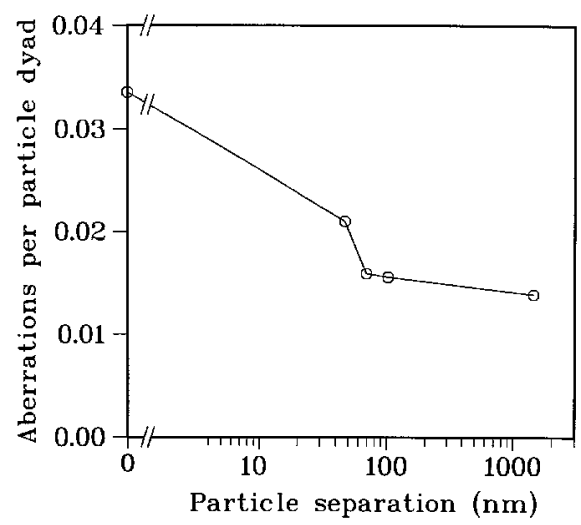

Figure 4. Yield of chromosome aberrations in V79 cells exposed to correlated pairs of hydrogen ions, as a function of the mean separation of the two ions. Data from Geard (1985). 
nanom eter dimensions, as the energy of low energy neutrons is decreased below $\sim 100 \mathrm{keV}$, their biological effectiven ess would be expected to decrease, since the LET of the recoil protons decreases. However, a series of experiments from Harwell (e.g. Edwards et al. 1990) have suggested that, as the neutron energy is decreased $<100 \mathrm{keV}$, the biological effectiveness does not decrease, again implying an enhancement in biological effect caused by proximity effects at the sub-micrometer level.

3.2.4. Varying LET. Our basic presumption is that exchange-type chromosomal aberrations result from pairs of energy depositions or, more generally, energy deposition clusters (Brenner and Ward 1992); under this presumption, any set of experiments measuring aberrations after exposure to a variety of radiations with different distributions of distances between energy deposition clusters can be considered as a probe of proximity effects: specifically of the separation-dependent probability that pairs of energy-deposition clusters result in a chromosomal aberration. Thus, for example, systematic experiments with charged particles of different LET, such as those of Todd, Barendsen, Skarsgard and coworkers, and others (surveys in Lloyd and Edwards 1983, Goodhead 1987) can potentially be interpreted as probes of proximity effects for various endpoints, both at the micrometer level (because of the differing LET of the radiations) and at the nanometer level (because of the differing delta ray spectra produced by the different charged particles).

\subsubsection{Quantitative modelling of experiments with} differing radiations. In an attempt to extract quantitative data on proximity effects, modeling has been applied to the soft X-ray experiments (Brenner and Zaider 1984, Brenner et al. 1987), the molecular ion experiments (Kellerer et al. 1980, Zaider and Brenner 1984), and RBE-LET experiments (Zaider and Rossi 1985, Brenner 1988). Typically, these quantitative models have used the Theory of Dual Radiation Action, in its distance formulation (Kellerer and Rossi 1978). In this approach it is assumed that pairs of dsbs (described as 'sublesions') interact with a distance dependent probability to produce aberrations (generically referred to as 'lesions'). As discussed in $\S 2$, the most common current view is that illegitimate reunion occurs between dsb-free ends, rather than dsbs, but this difference should not drastically affect the proximity estimates.

Typical results from such analyses are shown in

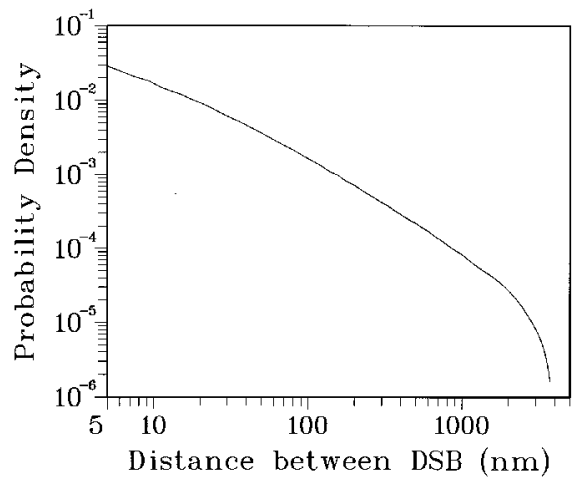

Figure 5. Estimated probability density that two energy deposition clusters capable of producing dsb ultimately produce a chromosomal aberration, as a function of the distance between the energy deposition clusters (derived from Brenner 1988). Note the strong estimated dependence on proximity.

Figure 5, which shows the probability density for production of an aberration as a function of the distance between the initial energy deposition clusters which produced the participating pair of dsbs. The marked distance dependence shown in Figure 5 represents strong, though model-dependent, evidence for proximity effects.

Figure 6, based on the same type of analysis, shows that aberrations formed by intra-track action are generally the result of pairs of breaks separated by short distances $(<100 \mathrm{~nm})$; increasing the dose increases the relative contribution of aberrations formed by inter-track action, which, as shown in Figure 6, generally result from pairs of breaks separated by distances $>100 \mathrm{~nm}$.

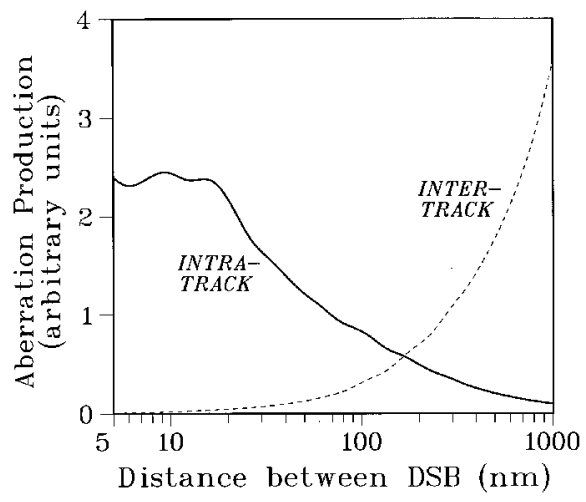

Figure 6. Results of a model analysis of the molecular ion experiment (based on Zaider and Brenner 1984). The height of the curves is proportional to the mean number of aberrations per cell originating in inter-track (dashed curve) or intra-track (solid curve) interactions between breaks that are formed a given distance apart. The relative normalization of the two curves corresponds to a dose of $0.3 \mathrm{~Gy}$. Smaller doses correspond to a smaller inter-track component, larger doses a larger one. 


\subsection{Inferences from dose-dependence}

As discussed above, proximity will differentially affect the yields of aberrations formed by in tratrack action relative to those formed through inter-track action. This is because dsbs from inter-track action are randomly located relative to one another through the cell nucleus, whereas those from intra-track action are typically closer together, reflecting the track structure of the radiation. From this premise, it can be seen that the ratio of aberrations formed by intra-track action to those formed by inter-track action can be used as a probe of proximity effects. In practice, this ratio is often estimated from the ratio $\alpha / \beta$ of a linear-quadratic fit to the dose-response curve, which, in a simple usage of the linear-quadratic model, can be interpreted as reflecting the ratio of the intra- to inter-track yields (Appendix 3). Using this approach for various radiations within the context of the Theory of Dual Radiation Action, estimates of maximum interactions distances are $\sim 1 \mu \mathrm{m}$ or somewhat less (survey in Bauchinger 1983).

\subsection{Analysis of variance}

Proximity effects have also been inferred from a large cell-to-cell variance in aberration production which is observed at high LET (e.g. Virsik and Harder 1981). Microdosimetric interpretations of the large variance have used site models. The idea is that the smaller a site, the larger the variance of specific energy (energy deposited per unit mass) for the sites, and therefore the larger the variance of aberration production. Thus a large variance in the number of dicentrics per cell can be interpreted as evidence for small sites, i.e. for proximity effects (survey in Brenner and Sachs 1994b). Some attempts to analyse this effect quantitatively have contained ambiguities as to whether the discussion concerns one small sensitive site within the nucleus, many sites which more or less fill out the nucleus, or some other configuration. If there is more than one site per cell nucleus, variance analyses for high LET must in principle take into account correlations between neighboring sites which occur when a primary radiation track passes through the nucleus; these correlations have never, as far as we know, been incorporated into variance models. An additional difficulty with drawing proximity inferences from variance measurements is that population inhomogeneities, e.g. cell sub-populations of different radiosensitivities, can increase observed variances even in the absence of LET effects (Couzin and Papworth 1979).

\section{Kinetics}

Although this review has emphasized timeindependent analyses of proximity effects, it is, of course, the case that chromosome aberration formation is dynamic. A great deal of work, experimental and theoretical, has been done on chromosome aberration kinetics. Particularly relevant on the experimental side are PCC data (recent surveys in Cornforth and Bedford 1993, Goodwin $e t$ al. 1994, Gray et al. 1994, Loucas and Geard 1994, Durante et al. 1996, Evans et al. 1996), low dose-rate studies (survey in Lloyd and Edwards 1983) and split-dose experiments (e.g. Greinert et al. 1996). Theoretical studies show that the details of how aberrations develop in time can influence the shape of dose response curves (Appendix 3). One PCC study reported dicentrics developing so rapidly after irradiation that the process is virtually completed with $\leqslant 1$ h (Vyas et al. 1991), but most indicate a more gradual rise for part or all of the dicentric yield (e.g. Greinert et al. 1995, Evans et al. 1996).

Most theoretical models of chromosome aberration kinetics (survey in Sachs et al. 1992) have neglected proximity effects, but some recent treatments incorporate such effects (e.g. Brenner 1990, Edwards et al. 1996, Moiseenko et al. 1996, $\mathrm{Wu}$ et al. 1996a). Thus, for example, one would expect that exchange-type aberrations formed by densely ionizing radiations might be formed, on average, more rapidly than by X-rays, due to the closer proximity of the free dsb ends involved (compare Figure 6). Assuming a model where illegitimate reunion competes kinetically with systematic restitution (rather than coming later as in the models of $\S 3$ ), Brenner (1990) showed that a consequence of the proximity-related difference in time development at different LET values is that differing numbers of aberrations will ultimately be produced by the same number of initially induced dsbs. This is because restitution has less time to occur when illegitimate reunion occurs more rapidly.

\section{Discussion}

\subsection{Summary}

We have discussed the influence of proximity 
effects on the production of chromosome aberrations by ionizing radiation. There are two main lines of evidence for proximity effects: (1) comparing different aberration types shows that there is a statistical bias for certain aberrations involving fewer chromosome relative to those involving more chromosomes, and for in tra-arm aberrations relative to inter-arm aberrations (see $\S 3.1$ ); (2) varying radiation quality and dose shows there is a statistical bias for aberrations caused by intratrack, closely bunched dsbs relative to aberrations caused by randomly scattered dsbs (see $\S \S 3.2$ and 3.3). Additional evidence comes from analysing the post-irradiation time evolution of aberrations (see $\S 4)$. Maximum interaction distances inferred from the data are approximately 1$2 \mu \mathrm{m}$ for inter-track, inter-chromosomal illegitimate reunions; for in tra-track or intra-chromosomal illegitimate reunions, considerably shorter distances predominate.

The analysis of proximity effects in $\S 3.1$ used comparisons of experimental results with results expected from randomness. Evidence for randomness was discussed in Appendix 1. To the extent that randomness holds, it gives a very powerful way to interrelate the frequencies of different aberration types.

\subsection{Interphase chromatin geometry and motion}

The aberration data indicate that chromosomes are localized within territories roughly $1 / 3$ the linear size of a cell nucleus (see $\S 3.1$ ), a conclusion in agreement with direct data (Appendix 2). Significant yields of complex chromosome aberrations (see $\$ 3.1 .2$ ) can be interpreted in one of two ways. Perhaps there is a large amount of overlapping and intertwining among chromosomes or chromosome stretches, either randomly (Brenner and Sachs 1994a), or at locations where chromatin is brought together for genetic or functional purposes (Savage 1996). Alternatively, after irradiation there could be considerable motion of each chromosome relative to other chromosomes. In order for free ends to find each other and undergo illegitimate reunion, one needs either highly organized motion (Savage 1996) or random motion which is almost volume-filling (i.e. a free end moves in a path so irregular that even a small sub-volume is likely to be crossed at least once). The whole issue of motion remains virtually unexplored territory (Appendix 2), and investigation of the way $F$ depends on LET should provide an informative probe for relative chromosome motion (see $\$ 3.1 .1$ ).

Proximity effects in aberration formation are a window on fundamental biology. An understanding of how and why aberrations occur, what a G1 chromatin fiber looks like overall, geometric interrelations of different chromosomes during cell cycle interphase, and chromosome motion during interphase can clarify the processes of DNA repair, DNA replication, transcription and differentiation.

\subsection{Implications}

The evidence presented in this review suggests that a realistic understanding of chromosome aberration formation mechanisms is not possible without a parallel understanding of proximity effects. Currently our understanding is mainly phenomenological. Hopefully, exploration of chromosomal motion in the interphase nucleus will eventually allow more mechanistic interpretations. Mechanistic approaches have important implications. For example, analysing doseresponse relations has traditionally relied on modelling, and modelling incorporating proximity effects suggests that the classic linear-quadratic dose-response curve may well be an oversimplification (Appendix 3). Since dose-response relations are frequently applied to fractionated radiotherapy and are almost universally applied to epidemiological analyses of radiation-induced carcinogenesis, understanding their shape mechanistically is central to applications of radiobiology.

\section{Acknowledgements}

Research partially sponsored by NIH grants CA63897 (R.K.S.) and ES07361 (D.J.B.), and NSF grant 9302704 (A.M.C.). Work performed under the auspices of the US DOE by LLNL under contract no. W-7405-Eng-48 (A.M.C.). We are grateful to M. Cornforth, A. A. Edwards, C. Geard, D. Goodhead, S. Knehr, J. N. Lucas, T. Radivoyevitch, J. R. K. Savage, E. Schmid, P. J. Simpson and J. Thacker for discussions.

\section{Appendix 1. Randomness}

Starting with the early investigations (e.g. Lea 1946), quantitative analyses of chromosome aberration frequencies have almost always assumed some randomness properties. As will 
now be discussed, experiments suggest two main randomness properties, one for induction of reactive dsbs and one for rejoining of $d s b$ free ends. Either randomness property could hold without the other.

\section{A.1.1. Randomness of dsb induction}

The first randomness property is that dsb induction by ionizing radiation is (approximately) independent of location in the genome. For example, the probability distribution for reactive dsbs in a particular chromosome arm would be Poisson, with a mean proportional to the length, in base pairs, of that arm. We are here interested in averages over DNA stretches of $>1 \mathrm{Mb}$, corresponding to the limit of resolution of the aberration assay; for example, whether randomness of $\mathrm{dsb}$ induction holds at the nucleosome level, i.e. for stretches as short as $\sim 200 \mathrm{bp}$, is not directly relevant.

Randomness of dsb induction is suggested by comparing aberrations involving different chromosomes, of different lengths, for the same irradiation conditions. Given randomness and low doses, involvement of two defined parts of the genome in an aberration should be proportional to the product of the relevant genomic lengths (Savage and Papworth 1982, Hlatky et al. 1992, Lucas et al. 1992). For example, suppose the total length of the chromosomes in a genome is $G \mathrm{Mb}$, and we paint yellow a subset having length $Y \mathrm{Mb}$ (e.g. both homologues of chromosome 1), with the rest of the genome, having length $G-Y \mathrm{Mb}$, counterstained blue. Aberration frequencies can be compared with frequencies scored using a different painting scheme in which length $Y^{\prime}$ is painted yellow. Then, assuming randomness and low does, the relative numbers of translocations or dicentrics involving a yellow and a blue chromosome are, on average, given by the proportion (Savage and Papworth 1982, Lucas et al. 1992).

$$
Y(G-Y): Y^{\prime}\left(G-Y^{\prime}\right)
$$

This equation can be understood as follows. The dose being low, suppose there are just two reactive $\mathrm{d}$ sbs in the genome. Randomness of dsb induction implies that the probability that the first dsb is on the yellow portion is $Y / G$, and that the probability that the second $\mathrm{dsb}$ is on the blue portion is $(G-Y) / G$. Then the probability for these two independent events to occur is the product $Y(G-Y) / G^{2}$. Similarly, the probability for the first $\mathrm{dsb}$ to be on the blue portion with the second on the yellow portion is $(G-Y) Y / G^{2}$. Adding, the total chance for a yellow-blue illegitimate reunion is proportional to $2 Y(G-Y)$. The corresponding argument for the case where the yellow portion has length $Y^{\prime}$ thus gives equation 2 . Equation 2 implies that the total average frequency of translocations or dicentrics in the whole genome is given by (Lucas et al. 1992) $A G^{2} /[2 Y(G-Y)]$ where $A$ is the observed average for translocations or dicentrics involving the painted and unpainted portion.

For higher doses equation 2 should still apply approximately to apparently simple two-way interchanges, as defined in the caption to Figure 2, if randomness of dsb induction holds. Equation 2 requires some modification at higher doses, because the formation of complex aberrations competes with the formation of simple aberrations, but Monte Carlo computer simulations (Chen et al. 1996) show that no major corrections are required for apparently simple two-way interchanges, assuming in vitro irradiation of human lymphocytes or fibroblasts with doses of $\leqslant 6 \mathrm{~Gy}$.

A number of papers have reported approximate agreement with the results of equation 2 or corresponding results for G-banded whole-genome scoring (e.g. Savage and Papworth 1982, Lucas et al. 1992, Ohtaki 1992, Sachs et al. 1993, Kovacs et al. 1994, Durante et al. 1996, and papers quoted in these references). Figure 7 compares results reported by Simpson and Savage (1996) to

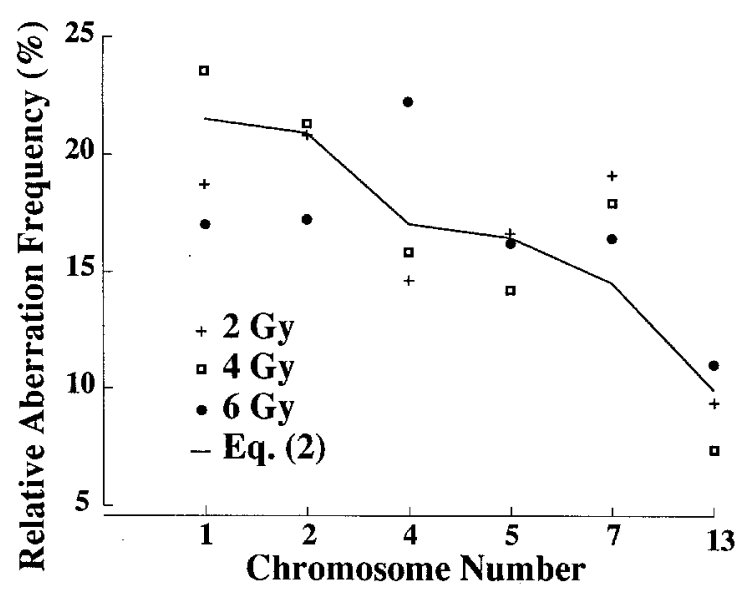

Figure 7. Aberration dependence on chromosome length. Data are from Savage and Simpson (1996, Table 1). Equation 2 is compared with the relative numbers of apparently simple interchanges (as defined in the caption to Figure 2) observed at three doses. The data shown involve a total of 1717 aberrations in 12531 cells (213 aberrations in 3791 cells at $2 \mathrm{~Gy}$, 964 aberrations in 6381 cells at $4 \mathrm{~Gy}$, and 564 aberrations in 2395 cells at $6 \mathrm{~Gy}$ ). 
equation 2. Except for chromosome 7, reasonable agreement between the data and the expectations based on randomness is found. There are also a number of papers which report deviations from equation 2 and caution has been urged (e.g. Jordan and Schwartz 1994, Natarajan et al. 1994, Schmid et al. 1995, Tanaka et al. 1996). In two thorough recent investigations, Knehr et al. (1994, 1996) report statistically significant differences between their observations and equation 2, with larger chromosomes generally participating in fewer exchanges than expected from equation 2, and smaller ones in more. However, if there are deviations from randomness there is no general pattern as to which chromosomes are over- or under-represented (Kovacs et al. 1994). For example, the results of Knehr et al. (1994, 1996), using lymphocytes, suggest that chromosome 7 participates in fewer translocations and dicentrics than expected from equation 2, whereas the results shown in Figure 7 for fibroblasts show an apparent excess instead. Similarly, FISH measurements showing under-representation of chromosome 1 for lym phocytes (Knehr et al. 1994, 1996; compare also Fernandez et al. 1995) are at variance with a number of G-banding studies on both lymphocytes and fibroblasts, and G-banding studies are themselves not consistent as to which chromosomes are under- and which are over-represented (Sachs et al. 1993, Kovacs et al. 1994). If there are systematic deviations from randomness of $\mathrm{dsb}$ induction, these systematic deviations cannot be very large.

An additional check on randomness of dsb induction can be obtained from the fact that, at low doses, randomness implies that the probability of an inter-arm exchange (centric ring or pericentric inversion; Figure $2 g$ and $h$ ) occurring in a particular chromosome is proportional to the product $L S$ of the lengths of the long arm $(L)$ and the short arm $(S)$ (Savage and Papworth 1982, Hlatky et al. 1992). The explanation of this prediction is analogous to the explanation, given above, of equation 2. One data set shows approximate agreement with the prediction (Sachs et al. 1993). Since these inter-arm exchanges are less frequent than dicentrics or translocations, the statistics which can be obtained are less convincing.

\section{A.1.2. Randomness of illegitimate rejoining}

The second randomness property is that, proximity effects apart, rejoining is random. For the breakage-and-reunion model this would mean that the probability a given free end rejoins with any other free end depends at most on their initial spatial separation, and that different illegitimate reunions do not influence each other.

One implication of random rejoining which is subject to direct experimental check is that apparently simple dicentrics (Figure 2b) should occur at the same frequency as apparently simple translocations (Figure 2c) (Lucas et al. 1996). This is essentially because the presence or absence of a centromere, which is usually many $\mathrm{Mb}$ distant from the dsb free ends, does not bias rejoining probabilities. Experimental checks of this equality have given inconsistent results, with some reports of near equality and other reports of a substantially higher number of translocations. Surveys of results on the translocation/dicentric ratio, from opposing perspectives, have been given by Knehr and coworkers $(1994,1996)$ and by Lucas et al. (1996).

The comparison of dicentrics and translocations has been somewhat confounded by scoring conventions different from laboratory to laboratory; if it is desired to check randomness directly, aberrations that are visibly complex in the painting scheme of Figure 2a-c should not be included in the comparison (Lucas et al. 1996). X-ray experiments on fibroblasts conducted by Savage and Simpson at MRC, using apparent simplicity as one scoring criterion so that visibly complex aberrations were not included in the comparison, gave 903 apparently simple translocations and 837 apparently simple dicentrics, for a ratio of 1.08 (Simpson, private communication, 1996). Approximate equality holds, and correction for background frequencies, which can be large in some cases (Griffin et al. 1995), would be expected to reduce the ratio closer to $1 \cdot 0$. One recent paper using this criterion also reports near-equality (Kanda and Hayata 1996).

Additional evidence on randomness of illegitimate reunions is found in other data. For example, for the doses specified in Table 1, simulations based on randomness predict, and the data show, near equality of the $2 \mathrm{~F}$ and $2 \mathrm{G}$ aberration types (Table 1). Similarly, centric rings and pericentric inversions appear to occur in approximately equal numbers (Savage and Papworth 1982), as do intra-arm inversions and acentric rings (Muhlmann-Diaz and Bedford 1995), as expected from randomness of illegitimate reunions. These additional checks involve smaller numbers of aberrations so they are less robust than observations comparing apparently simple dicentrics and translocations. 


\section{A.1.3. Summary}

It appears that both randomness properties are good first approximations, and can be used as idealizations to get a general overview of the data, even though they may not hold exactly. Assuming randomness allows systematic comparisons of results obtained using different FISH painting schemes of any kind, systematic comparisons of FISH scoring to older, whole-genome scoring, and systematic intercomparisons of the whole spectrum of simple or complex observed aberration types. Equations 1 and 2 furnish examples of how comparisons can be made, but in practice, especially for complex aberrations and/ or high doses, Monte Carlo computer simulations are needed for the comparisons (Chen et al. 1996).

\section{Appendix 2. Direct studies of chromosome geometry and motion}

Chromosome aberration studies and direct investigations of large-scale chromatin geometry are complementary. Aberration yields are influenced by, and act as probes for, chromosome geometry and motion during $\mathrm{G}_{0} / \mathrm{G}_{1}$. During cell cycle interphase, a chromosome consists of a long chromatin fiber, containing not only DNA but also a number of tightly associated proteins (Tsanev et al. 1993). Because of the aberration assay's limit of resolution, the scales of interest in the present context are large, from about $0 \cdot 1 \mathrm{Mb}$ on up to the full length of a human chromosome, which for chromosome 1 is almost $300 \mathrm{Mb}$.

At smaller scales, very detailed geometric information is available (Tsanev et al. 1993): the basic double helix has a scale of about $10 \mathrm{bp}$, nucleosomes have a scale of several hundred, the $30-\mathrm{nm}$ chromatin fiber has a scale of several thousand, and there are chromatin loops, averaging perhaps $60000 \mathrm{bp}$. The influence of such geometric structures on ionizing radiation damage has often been discussed. But for analysing chromosome aberrations, even the largest of these structures, the $0.06 \mathrm{Mb}$ loops, are too small to play a central role. For more than 3 orders of magnitude, from $\sim 0 \cdot 1$ to $\sim 300 \mathrm{Mb}$, the difficulty of tracking chromatin as it winds and twists its way through the interphase nucleus long precluded finding data sufficient for quantitative geom etric modelling.

Direct observations of interphase chromosomes, primarily observations using FISH, are now furnishing such data (Lichter et al. 1988, surveys in Cremer et al. 1993, Yokota et al. 1995). The main results may be summarized as follows. the bulk of any one chromosome during $\mathrm{G}_{0} / \mathrm{G}_{1}$ is localized in an irregular 'territory' or 'domain' whose diameter is very roughly $1 / 3$ the nuclear diameter. There may be, and probably are, 'wisps', perhaps consisting of little more than a single chromatin loop, which emanate from the territory. On scales of $\geqslant 0 \cdot 1 \mathrm{Mb}$ the chromatin may be approximately a random walk, modulated by systematic folding at the scale of several Mb (Sachs et al. 1995, Yokota et al. 1995). Visually there often appears to be little overlap between territories of different chromosomes (Muenkel et al. 1996, Tanaka et al. 1996, and references there), but the degree of overlap is not yet known. There do not seem to be any systematic associations between different chromosomes during interphase, other than the specific chromosomes which are involved in the nucleolus.

As far as chromosome motion is concerned, even less is known for certain. There is some evidence for overall motions on a time scale of an $\leqslant 1$ h (De Boni 1994). There must be Brownian motion in various modes, but this is little studied and could be complicated. For example (Doi and Edwards 1988) there could be reptation or normal mode motion for a long polymer, hindered by the nuclear skeleton, by other chromosomes, or by other macro-molecules in the nucleus. Brownian motion in the normal modes of a polymer chain has the characteristics required to be compatible with the aberration data, namely rapid, wellmixing motion at small scales and slower motion at larger scales (Doi and Edwards 1988). There could also be some much more organized motion, choreographed by the cell nucleus for purposes such as transcription or repair (Cremer et al. 1993, Savage 1996).

\section{Appendix 3. Dose dependence of aberration frequencies}

Understanding the shape of the dose-response curve for the formation of chromosome aberrations goes hand in hand with understanding the corresponding aberration formation mechanisms. We make here some comments on the linearquadratic dose-response relation for exchangetype aberrations, alternative dose-response relations, and the influence of proximity effects on the shape of dose-response relations.

\section{A.3.1. The linear-quadratic equation}

The yield of exchange-type chromosomal 
aberrations has often been described by the linear-quadratic (LQ) relationship between yield and dose:

$$
\text { average yield }=\alpha D+\beta D^{2} .
$$

Following Sax (1940) and Lea and Catcheside (Lea 1946), the two terms in the LQ equation have generally been interpreted as follows: (i) the linear term $(\alpha D)$ gives the yield of aberrations from intra-track action, involving chromosomal breaks induced by a single primary radiation track; and (ii) the quadratic term $\left(\beta D^{2}\right)$ gives the yield of aberrations from inter-track action, involving two chromosomal breaks induced by two different, independent primary radiation tracks. Because breaks induced by the same primary track tend to be closer together than breaks resulting from different primary tracks, proximity effects are generally most important for the in tratrack term $\alpha D$, and enhance it relative to the intertrack term $\beta D^{2}$.

Clearly, the linear term $(\alpha D)$ in equation 3 will dominate at low doses, and essentially all mechanistic models of aberration production in the literature agree with this prediction: that the yield of radiation-produced aberrations at very low doses will change linearly with dose, the coefficient being a measure of intra-track action. The quadratic term $\left(\beta D^{2}\right)$ will dominate at higher doses, with $\alpha / \beta$ being the dose at which the contributions to the yield of these two components are equal. Generally speaking, at low LET, except for very low doses (Bauchinger 1995), the $\beta D^{2}$ term may dominate, whereas for most high LET experiments the $\alpha D$ term is dominant over the entire dose-range used.

\section{A.3.2. Deviations from the linear-quadratic equation}

Many aberration endpoints, such as average number of dicentrics per cell, show 'saturation', i.e. at large doses their yield is reduced because of competition from other aberration types and/or because there are only a limited number of centromeres in a genome (survey in Savage 1996). Saturation can cause large deviations from $L Q$ behaviour (Norman and Sasaki 1966). However, there are endpoints which are not subject to saturation, e.g. the per-cell number of illegitimate reunions (painted or not), which generalizes the colour junction number used by Tucker et al. (1996b). For example, the number of illegitimate reunions in Figure $2 \mathrm{~b}$ or $\mathrm{c}$ is two, and in Figure 2e it is three. Yield of illegitimate reunions does not show saturation effects because it is sensitive to all forms of exchange-type aberrations, including complex aberrations.

It is often taken for granted that for an endpoint not subject to saturation any pairwise interaction model (such as the breakage-and-reunion model or the alternate model of $\S 2.5$ ) automatically leads to a quadratic dependence of yield on dose for high doses, as in equation 3. However, as Lea himself emphasized (Lea 1946, pp. 256, 262-6) this assumption is warranted only if pairwise misrepair (such as illegitimate reunion) competes kinetically with repair (such as restitution) and occurs only in a small fraction of cases. There are various other possible forms of kinetics (Hahnfeldt et al. 1992), and for some kinds of kinetics the use of the $L Q$ equation is not warranted (Lea 1946, Read 1965, Sachs and Brenner 1993).

\section{A.3.3. An $L Q L$ dose-response relation}

In the breakage-and-reunion model as described in $\S 2.2$, illegitimate reunion does not compete kinetically with systematic restitution, and the $L Q$ dependence on dose, equation 3, does not hold, even for a non-saturating yield such as total illegitimate reunion number. Assuming, as is reasonable, that the average number of $\mathrm{dsbs}$ prior to systematic restitution is linear in dose and that the choice between systematic restitution or activation follows linear kinetics, the average number of reactive dsbs is linear in dose, not quadratic. The dose dependence of the yield will then have three different regimes (Figure 8). At small doses, the yield will be linear in dose due to intra-track action, as discussed above. At intermediate doses, there will be a roughly quadratic regimen, corresponding to inter-track action with the number of reactive dsbs so small that accidental restitution competes effectively with illegitimate reunion. Finally, at still higher doses, free ends from most of the reactive dsbs will undergo illegitimate reunion rather than accidental restitution and this will lead to an approximately linear (not quadratic) increase of illegitimate reunions with dose. Over-all one has a $L Q L$ (linear-quadraticlinear) type of dose-response.

Proximity effects favour accidental restitution over illegitimate reunion. For example, in a site model, the number of reactive dsbs per site is smaller when the number of sites is large, implying that accidental restitution occurs more frequently for a given number of illegitimate reunions. Proximity effects therefore extend the 


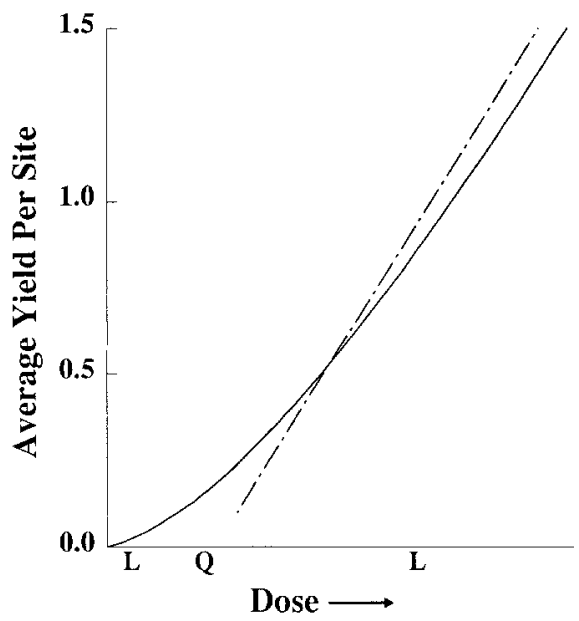

Figure 8. Schematic, illustrating that pairwise interaction does not always result in a quadratic yield at large doses. The vertical axis specifies yield, measured in average number of illegitimate reunions per site (see text). The solid curve was calculated by adding a term of the form $\alpha D$, corresponding to intra-track action important at low doses, to the yield calculated by the breakage-and-reunion model described in $\$ 2.2$. For low doses, the curve has a predominantly linear portion (indicated by L); as the dose increases there is a curvilinear, almost quadratic portion (indicated by Q); for still larger doses, however, the curve ultimately becomes almost linear again (indicated by L), asymptotically approaching the straight line shown dashed. The dose axis is in arbitrary units and $\alpha$ was chosen for convenience. However, the scale of the vertical axis is intrinsic, being uniquely determined by the model.

mid-range, nearly quadratic portion of the doseresponse relation (Figure 8) to higher doses. Thus the observed quadratic component in the dose-response relation for dicentrics at low LET need not imply reaction kinetics in which illegitimate reunion competes with systematic restitution. It may reflect proximity effects combined with reaction kinetics in which illegitimate reunion occurs only after each dsb has committed either to systematic restitution or to becoming reactive (Figure 8 ).

In fact there is recent evidence from FISH experiments (Simpson and Savage 1996) that, at high doses, the yield of simple aberrations may not increase as rapidly as a quadratic relationship with dose would predict, which could be consistent with saturation or with the $L Q L$ type of dose-response relation shown in Figure 8.

\section{References}

Ballard, S. G. and WARD, D. C., 1993, Fluorescence in situ hybridization using digital imaging microscopy. Journal of Histochemistry and Cytochemistry, 41, 1755-1759.
Bauchinger, M., 1983, Microdosimetric aspects of the induction of chromosome aberrations. In Radiation-Induced Chromosome Damage in Man, edited by: T. Ishihara and M. S. Sasaki (New York: Alan R. Liss), pp. 1-22.

Bauchinger, M., 1995, Quantification of low-level radiation exposure by conventional chromosome aberration analysis. Mutation Research, 339, 177-189.

Bender, M. A., Awa, A. A., Brooks, A. L., Evans, H. J., Groer, P. G., Littlefield, L. G., Pereira, C., Preston, R. J. and Wachholz, B. W., 1988, Current status of cytogenetic procedures to detect and quantify previous exposures to radiation. Mutation Research, 196, 103-159.

BRENNER, D. J., 1988, On the probability of in teraction between elementary radiation-induced chromosomal injuries. Radiation and Environmental Biophysics, 27, 189-199.

Brenner, D. J., 1990, Track structure, lesion development, and cell survival. Radiation Research, 124, S29-37.

Brenner, D. J., 1996, Direct biological evidence for significant neutron dose to survivors of the Hiroshima atomic bomb. Radiation Research, 145, 653-653.

Brenner, D. J., Bird, R. P., Zaider, M., Goldhagen, P., Kliauga, P. J. and Rossi, H. H., 1987, Inactivation of synchronized mammalian cells with low-energy $\mathrm{X}$ rays-results and significance. Radiation Research, 110, 413-427.

Brenner, D. J. and SAChs, R. K., 1994a, Chromosomal 'fingerprin ts' of prior exposure to densely-ionizing radiation. Radiation Research, 140, 134-142.

Brenner, D. J. and Sachs, R. K., 1994b, Generalized microdosimetric calculations of cell-to-cell variance. Radiation Protection Dosimetry, 52, 21-24.

Brenner, D. J. and Sachs, R. K., 1996a, Comments on 'Comment on the ratio of chromosome-type dicentric interchanges to centric rings for track-clustered compared to random breaks' by Savage and Papworth. Radiation Research, 146, 241-242.

Brenner, D. J. and SAChs, R. K., 1996b, Response to 'Comments on "Direct Biological Evidence for a Significant Neutron Dose to Survivors of the Hiroshima Atomic Bomb", by E. Schmid and M. Bauchinger. Radiation Research. 146, 481-482.

Brenner, D. J. and WARD, J. F., 1992, Constraints on energy deposition and target size of multiply damaged sites associated with DNA double-strand breaks. International Journal of Radiation Biology, 61, 737-748.

Brenner, D. J., WARD, J. and SACHS, R. K., 1994, Track structure, chromosome geometry and chromosome aberrations. In Basic Life Sciences. 63: Computational Approaches in Molecular Radiation Biology, edited by: M. N. Varma and A. Chatterjee (Plenum Press, New York), pp. 93113.

Brenner, D. J. and Zaider, M., 1984, Modification of the theory of dual radiation action for attenuated fields, II. Application to the analysis of soft x-ray results. Radiation Research, 99, 492-501.

Brown, J. M., Evans, J. W. and Kovacs, M. S., 1993, Mechanism of chromosome exchange formation in human fibroblasts: insights from 'chromosome painting'. Environmental and Molecular Mutagenesis, 22, 218-24.

Brown, J. M. and Kovacs, M. S., 1993, Visualization of nonreciprocal chromosome exchanges in irradiated human fibroblasts by fluorescent in situ hybridization. Radiation Research, 136, 71-76.

Chadwick, K. H. and Leenhouts, H. P., 1981, The Molecular Theory of Radiation Biology (Berlin: Springer).

Chen, A. M., Lucas, J. N., Hill, F. S., Brenner, D. J. and Sachs, R. K., 1996, Proximity effects for chromosome 
aberrations measured by FISH. Internation al Journal of Radiation Biology, 69, 411-420.

Cornforth, M. N., 1990, Testing the notion of the one-hit exchange. Radiation Research, 121, 21-27.

Cornforth, M. N. and Bedford, J. S., 1987, A quantitative comparison of potentially lethal damage repair and the rejoining of interphase chromosome breaks in low passage normal human fibroblasts. Radiation Research, 111, 385-405.

Cornforth, M. N. and Bedford, J. S., 1993, Ionizing radiation damage and its early development in chromosomes. In Advances in Radiation Biology, vol. 17. DNA and Chromatin Damage Caused by Radiation, edited by J. T. Lett and W. K. Sinclair (San Diego: Academic), pp. 423-496.

Couzin, D. and PAPworth, D. G., 1979, The over-dispersion between cells of chromosomal aberrations. Journal of Theoretical Biology, 80, 249-258.

Cremer, T., Kurz, A., Zirbel, R., Dietzel, S., Rinke, B., Schrock, E., Speicher, M. R., Mathieu, U., Jauch, A., Emmerich, P., Schertan, H., Ried, T., Cremer, C. and Lichter, P., 1993, Role of chromosome territories in the functional compartmentalization of the cell nucleus. Cold Spring Harbor Symposia on Quantitative Biology, 58, 777-792.

DE Boni, U., 1994, The interphase nucleus as a dynamic stucture. International Review of Cytology, 150, 149-171.

Dor, M. and Edwards, S. F., 1988, The Theory of Polymer Dynamics (Oxford: Oxford University Press).

Durante, M., George, K. and Yang, T. C., 1996, Biological dosimetry by interphase chromosome painting. Radiation Research, 145, 53-60.

Edwards, A. A., Lloyd, D. C. and Prosser, J. S., 1990, The induction of chromosome aberrations in human lymphocytes by $24 \mathrm{keV}$ neutrons. Radiation Protection Dosimetry, 31, 265-268.

Edwards, A. A., Moiseenko, V. V. and Nikjoo, H., 1996, On the mechanism of the formation of chromosomal aberrations by ionizing radiation. Radiation and Environmental Biophysics, 35, 25-30.

Evans, J. W., LiU, X. F., Kirchgessner, C. U. and Brown, J. M., 1996, Induction and repair of chromosome aberrations in scid cells measured by premature chromosome condensation. Radiation Research, 145, 39-46.

Fernandez, J. L., Campos, A., Goyanes, V., Losada, C., Veiras, C. and Edwards, A. A., 1995, X-ray biological dosimetry performed by selective painting of human chromosomes 1 and 2. International Journal of Radiation Biology, 67, 295-302.

Finnon, P., Lloyd, D. C. and Edwards, A. A., 1995, Fluorescence in situ hybridization detection of chrom osomal aberrations in human lymphocytes - applicability to biological dosimetry. International Journal of Radiation Biology, 68, 429-435.

GEARD, C. R., 1985, Charged particle cytogenetics: effects of LET, fluence, and partial separation on chromosome aberrations. Radiation Research, 104, S112-121.

Gebhart, E., Neubauer, S., Schmitt, G., Birkenhake, S. and Dunst, J., 1996, Use of a three-color chromosome in situ suppression technique for the detection of past radiation exposure. Radiation Research, 145, 47-52.

Goodhead, D. T., 1987, Relationship of microdosimetric techniques to applications in biological systems. In The Dosimetry of Ionizing Radiation, vol. II, edited by K. Kase, B. Bjarngard and F. Attix (Orlando: Academic), pp. 1-89.

Goodhead, D. T., 1994, Initial events in the cellular effects of ionizing radiations: clustered damage in DNA. International Journal of Radiation Biology, 65, 7-17.
Goodhead, D. T., Thacker, J. and Cox, R., 1993, Weiss Lecture. Effects of radiations of different qualities on cells: molecular mechanisms of damage and repair. International Journal of Radiation Biology, 63, 543-556.

Goodwin, E. H., Blakely, E. A. and Tobias, C. A., 1994, Chromosomal damage and repair in $\mathrm{G}_{1}$-phase Chinese hamster ovary cells exposed to charged-particle beams. Radiation Research, 138, 343-351.

Gray, J. W., Pinkle, D. and Brown, J. M., 1994, Fluorescence in situ hybridization in cancer and radiation biology. Radiation Research, 137, 275-289.

Greinert, R., Detzler, E., Volkmer, B. and Harder, D., 1995, Kinetics of the formation of chromosome aberrations in X-irradiated human lymphocytes: analysis by premature chromosome condensation with delayed fusion. Radiation Research, 144, 190-197.

Greinert, R., Volkmer, B., Virsik-Peuckert, R. P. and Harder, D., 1996, Comparative study of the repair kinetics of chromosomal aberrations and DNA strand breaks in proliferating and quiescent $\mathrm{CHO}$ cells. International Journal of Radiation Biology, 70, 33-43.

Griffin, C. S., Marsden, S. J., Stevens, D. L., Simpson, P. J. and Savage, J. R. K., 1995, Frequencies of complex chromosoome exchange aberrations induced by ${ }^{238} \mathrm{Pu}$ $\alpha$-particles and detected by fluorescence in situ hybridization using single chromosome-specific probes. International Journal of Radiation Biology, 67, 431-439.

Hahnfeldt, P., Hlatky, L. R., Brenner, D. J. and Sachs, R. K., 1995, Chromosome aberrations produced by radiation: the relationship between excess acentric fragments and dicen trics. Radiation Research, 141, 136-152.

Hahnfeldt, P., Sachs, R. K. and Hlatky, L. R., 1992, Evolution of DNA damage in irradiated cells. Journal of Mathematical Biology, 30, 493-511.

Hlatky, L. R., Sachs, R. K. and Hahnfeldt, P., 1992, The ratio of dicentrics to centric rings produced in human lymphocytes by acute low-LET radiation. Radiation Research, 129, 304-308.

Jones, L. A., Glegg, S., Edwards, S., Kent, C., Peacock, J. H. and Steel, G. G., 1995, The relationship between cell death and residual radition-induced damage. In Radiation Research 1895-1995, Congress Proceedings, vol. I, edited by U. Hagen, H. Jung and C. Streffer (Wurzburg: Wurzburg University Press), p. 380.

Jordan, R. and Schwartz, J. L., 1994, Noninvolvement of the X chromosome in radiation-induced chromosome translocations in the human lymphoblastoid cell line TK6. Radiation Research, 137, 290-4.

KANDA, R. and Hayata, I., 1996, Comparison of the yields of translocations and dicentrics measured using conventional giemsa staining and chromosome painting. International Journal of Radiation Biology, 69, 701-705.

Kellerer, A. M., 1985, Fundamentals of Microdosimetry. In The Dosimetry of Ionizing Radiation, vol. I, edited by $\mathrm{K}$. Kase, B. Bjarngard and F. Attix (Orlando: Academic), pp. $77-162$.

Kellerer, A. M., Lam, Y.-M. P. and Rossi, H. H., 1980, Biophysical studies with spatially correlated ions. IV. Analysis of cell survival data for diatomic deuterium. Radiation Research, 83, 511-528.

Kellerer, A. M. and Rossi, H. H., 1978, A generalized formulation of dual radiation action. Radiation Research, 75, $471-488$.

Knehr, S., Zitzelsberger, H., Braselmann, H. and Bauchinger, M., 1994, Analysis for DNA-proportional distribution of radiation-induced chromosome aberrations in 
various triple combinations of human chromosomes using fluorescence in situ hybridization. International Journal of Radiation Biology, 65, 683-690.

Knehr, S., Zitzelsberger , H., Braselmann, H., Nahrstedt, U. and Bauchinger, M., 1996, Chromosome analysis by fluorescence in situ hybridization: further indications for a nonDNA-proportional involvement of single chromosomes in radiation-induced structural aberrations. International Journal of Radiation Biology, 70, 385-392.

Kovacs, M. S., Evans, J. W., Johnstone, I. M. and Brown, J. M., 1994, Radiation-induced damage, repair and exchange formation in different chromosomes of human fibroblasts determined by fluorescence in situ hybridization. Radiation Research, 137, 34-43.

LEA, D. E., 1946, Actions of Radiations on Living Cells (London: Cambridge University Press).

Lichter, P., Cremer, T., Borden, J., Manuelidis, L. and Ward, D. C., 1988, Delineation of individual human chromosomes in metaphase and interphase cells by in situ suppression hybridization using recombinant DNA libraries. Human Genetics, 80, 224-234.

Loucas, B. D. and GEARD, C. R., 1994, Initial damage in human interphase chromosomes from alpha particles with linear energy transfers relevant to radon exposure. Radiation Research, 139, 9-14.

Lloyd, D. C. and Edwards, A. A., 1983, Chromosome aberrations in human lymphocytes: effect of radiation quality, dose, and dose rate. In Radiation-Induced Chromosome Damage in Man, edited by: T. Ishihara and M. S. Sasaki (New York: Alan R. Liss) pp. 23-49.

Lucas, J. N., Awa, A., Straume, T., Poggensee, M., Kodama, Y., Nakano, M., Ohtaki, K., Weier, H. U., Pinkel, D., Gray, J. and Littlefield, G., 1992, Rapid translocation frequency analysis in humans decades after exposure to ionizing radiation. International Journal of Radiation Biology, 62, 53-63.

Lucas, J. N., Chen, A. M. and Sachs, R. K. 1996, Theoretical predictions on equality of radiation-produced dicen trics and translocations detected by chromosome painting. International Journal of Radiation Biology, 69, $145-153$.

LuCAS, J. N. and SACHS, R. K., 1993, Using 3-colour chromosome painting to decide between chromosome aberration models. Proceedings of the National Academy of Sciences, USA, 90, 1484-1487.

Moiseenko, V. V., Edwards, A. A. and Nikjoo, H., 1996, Modelling the kinetics of chromosome exchange formation in human cells exposed to ionizing radiation. Radiation and Environment Biophysics, 35, 31-35.

Muenkel, C., Eils, R., Imhoff, J., Dietzel, S., Cremer, C. and Cremer, T. 1996, Simulation of the distribution of chromosome targets in cell nuclei under topological constraints. Bioimaging, submitted.

Muhlmann-Diaz, M. C. and Bedford, J. S., 1995, Comparison of gamma-ray-induced chromosome ring and inversion frequencies. Radiation Research, 143, 175-180.

Natarajan, A. T., Balajee, A. S., Boei, J. J., Chatterjee, S., Darroudi, F., Grigorova, M., Noditi, M., Oh, H. J., Slijepcevic, P. and Vermeulen, S., 1994, Recent developments in the assessment of chromosomal damage. International Journal of Radiation Biology, 66, 615-623.

Neary, G. J., Savage, J. R. K. and Evans, H. J., 1964, Chromatid aberrations in Tradescantia pollen tubes induced by monochromatic X-rays of quantum energy 3 and $1.5 \mathrm{keV}$. International Journal of Radiation Biology, 8, 1-19.
Norman, A. and Sasaki, M. S., 1966, Chromosome-exchange aberrations in human lymphocytes. International Journal of Radiation Biology, 11, 321-328.

OHtaki, K., 1992, G-banding analysis of radiation-induced chromosome damage in lymphocytes of Hiroshima A-bomb survivors. Japanese Journal of Human Genetics, 37, 245-262.

Pandita, T. K., Pathak, S. and Geard, C. R., 1995, Chromosome end associations, telomeres and telomerase activity in ataxia telangiectasia cells. Cytogenetics and Cell Genetics, 71, 86-93.

Pohl-Ruling, J., Fscher, P., Lloyd, D. C., Edwards, A. A., Natarajan, A. T., Obe, G., Buckton, K. E., Bianchi, N. O., van Buul, P. P., Das, B. C. et al., 1986, Chromosomal damage induced in human lymphocytes by low doses of D-T neutrons. Mutation Research, 173, 267-272.

Raввіттs, T. H., 1994, Chromosomal translocations in human cancer. Nature, 372, 143-149.

READ, J., 1965, The induction of chromosome exchange aberrations by ionizing radiations: the 'site' concept. International Journal of Radiation Biology, 9, 53-65.

Revell, S. H., 1974, The breakage-and-reunion theory and the exchange theory for chromosomal aberrations induced by ionizing radiation: a short history. Advances in Radiation Biology, 4, 367-416.

Russell, N. S., Arlett, C. F., Bartelink, H. and Begg, A. C., 1995, Use of fluorescence in situ hybridization to determine the relationship between chromosome aberrations and cell survival in eight human fibroblast strains. International Journal of Radiation Biology, 68, $185-96$.

Sachs, R. K., Awa, A., Kodama, Y., Nakano, M., Ohtaki, K. and Lucas, J. N., 1993, Ratios of radiation-produced chromosome aberrations as indicators of large-scale DNA geometry during interphase. Radiation Research, 133, 345-350.

SAChS, R. K. and Brenner, D. J., 1993, Effect of LET on chromosomal aberration yields. I. Do long-lived, exchange-prone double strand breaks play a role? International Journal of Radiation Biology, 64, 677-688.

Sachs, R. K., Chen, P.-L., Hahnfeldt, P. and Hlatky, L. R., 1992, DNA damage caused by ionizing radiation. Mathematical Biosciences, 112, 271-303.

Sachs, R. K., Yokota, H., van-den-Engh, G., Trask, B. and Hearst, J. E., 1995, A random-walk/giant loop model for in terphase chromosomes. Proceedings of the National Academy of Sciences, USA, 92, 2710-2714.

SAVAGE, J. R. K., 1970, Sites of radiation induced chromosome exchanges. In Current Topics in Radiation Research, VI, edited by M. Ebert and A. Howard (London: North Holland), pp. 130-194.

SAVAGE, J. R. K., 1975, Radiation-induced chromosomal aberrations in the plant Tradescantia: dose-response curves, I. Prelim inary considerations. Radiation Botany, 15, $87-140$.

Savage, J. R. K., 1996, Insight into sites. Mutation Research.

Savage, J. R. K. and PAPworth, D. G., 1973, The relation ship of radiation-induced yield to chromosome arm number. Mutation Research, 19, 139-143.

Savage, J. R. K. and Papworth, D. G., 1982, Frequency and distribution studies of asymmetrical versus symmetrical chromosome aberrations. Mutation Research, 95, 7-18.

Savage, J. R. K. and Papworth, D. G. 1996, Comment on the ratio of chromosome-type dicentric interchanges to centric rings for track-clustered compared to random breaks. Radiation Research, 146, 236-240. 
Savage, J. R. K. and Simpson, P. J., 1994, FISH 'painting' patterns resulting from complex exchanges. Mutation Research, 312, 51-60.

SAx, K., 1940, An analysis of X-rays induced chromosomal aberrations in Tradescantia. Genetics, 25, 41-68.

Schmid, E. and Bauchinger, M., 1996, Comments on 'Direct Biological Evidence for a Significant Neutron Dose to Survivors of the Hiroshima Atomic Bomb'. Radiation Research, 146, 479-481.

Schmid, E., Braselmann, H. and Nahrstedt, U., 1995, Comparison of gamma-ray induced dicentric yields in human lymphocytes measured by conventional analysis and FISH. Mutation Research, 348, 125-30.

Simpson, P. J. and Savage, J. R. K., 1996, Dose-response curves for simple and complex chromosome aberrations induced by X-rays and detected using fluorescence in situ hybridization. International Journal of Radiation Biology, 69, 429-436.

Tanaka, K., Polp, S., Fischer, C., van Kaick, G., Kamada, N., Cremer, T. and Cremer, C., 1996, Chromosome aberrations in atomic bomb survivors and Thorotrast patients using two- and three-color chromosome painting of chrom osomal subsets. International Journal of Radiation Biology, 70, 95-108.

Thacker, J., Wilkinson, R. E. and Goodhead, D. T., 1986, The induction of chromosome exchange aberrations by carbon ultrasoft X-rays in V79 hamster cells. International Journal of Radiation Biology, 49, 645-656.

Tsanev, R., Russev, G., Pashev, I. and Zlatanova, J., 1993, Replication and Transcription of Chromatin (Boca Raton: CRS Press).

Tucker, J. D., Lee, D. A. and Moore, D. H. II, $1995 \mathrm{~b}$. Validation of chromosome painting. II. A detailed analysis of aberrations following high doses of ionizing radiation in vitro. International Journal of Radiation Biology, 67, 19-28.

Tucker, J. D., Morgan, W. F., Awa, A. A., Bauchinger, M., Blakely, D., Cornforth, M. N., Littlefield, L. G.,
Natarajan, A. T. and Shasserre, C., 1995a, PAINT: a proposed nomenclature for stuctural aberrations detected by whole chromosome painting. Mutation Research, 347, 21-24.

Virsik, R. P. and HARDER, D., 1981, Statistical in terpretation of the overdispersed distribution of radiation-induced dicen tric chromosome aberrations at high LET. Radiation Research, 85, 13-23.

Vyas, R. C., Darroudi, F. and Natarajan, A. T., 1991, Radiationinduced chromosomal breakage and rejoining in interphase-metaphase chromosomes of human lymphocytes. Mutation Research, 249, 29-35.

Wlodek, D. and Hittelman, W. N., 1988, The relationship of DNA and chromosome damage to survival of synchronized X-irradiated L5178Y cells. II. Repair. Radiation Research, 115, 566-573.

Wu, H., Durante, M., George, E., Goodwin, E. H. and Yang, T. Y., 1996a, Rejoining and misrejoining of radiationinduced chromatin breaks. 2. Biophysical model. Radiation Research, 145, 281-288.

Wu, H., Durante, M., Sachs, R. K. and Yang, T. Y., 1996b, A random walk model for in terphase chromosomes and the relationship between centric rings, acentric rings and excess acentric fragments induced by low-LET radiation. International Journal of Radiation Biology (submitted).

Yokota, H., van den Engh, G., Hearst, J. E., Sachs, R. K. and Trask, B., 1995, Evidence for the organization of chromatin in megabase pair-sized loops arranged along a random walk path in the human $G_{0} / G_{1}$ interphase nucleus. Journal of Cell Biology, 130, 1239-1249.

Zaider, M. and Brenner, D. J., 1984, The application of track calculations to radiobiology. III. Analysis of the molecular beam experiment results. Radiation Research, 100, 213-221.

ZAider, M. and Rossi, H. H., 1985, Dual radiation action and the initial slope of survival curves. Radiation Research, 104, S68-76. 